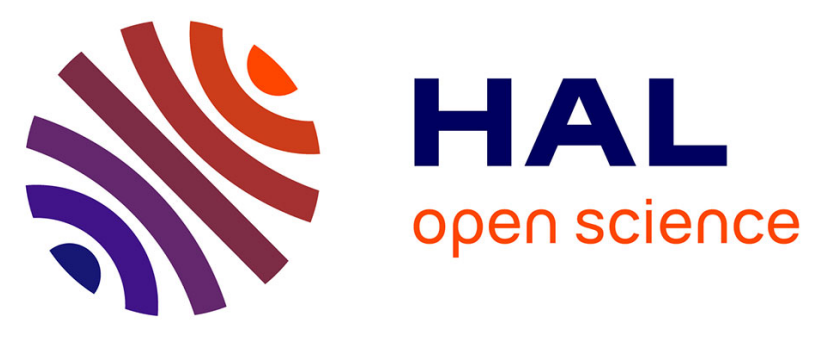

\title{
Second Harmonic Generation to Monitor the Interactions of the Antimicrobial Mycosubtilin with Membrane-Mimicking Interfacial Monolayers
}

Mehmet Nail Nasir, Emmanuel Benichou, Jean-Sebastien Guez, Philippe Jacques, Pierre-François Brevet, Françoise Besson

\section{To cite this version:}

Mehmet Nail Nasir, Emmanuel Benichou, Jean-Sebastien Guez, Philippe Jacques, Pierre-François Brevet, et al.. Second Harmonic Generation to Monitor the Interactions of the Antimicrobial Mycosubtilin with Membrane-Mimicking Interfacial Monolayers. BioNanoScience, 2012, 2 (2), pp.108-112. 10.1007/s12668-012-0037-6 . hal-02941901

\author{
HAL Id: hal-02941901 \\ https://hal.science/hal-02941901
}

Submitted on 17 Sep 2020

HAL is a multi-disciplinary open access archive for the deposit and dissemination of scientific research documents, whether they are published or not. The documents may come from teaching and research institutions in France or abroad, or from public or private research centers.
L'archive ouverte pluridisciplinaire HAL, est destinée au dépôt et à la diffusion de documents scientifiques de niveau recherche, publiés ou non, émanant des établissements d'enseignement et de recherche français ou étrangers, des laboratoires publics ou privés. 


\title{
Second harmonic generation to monitor the interactions of the antimicrobial
}

\section{mycosubtilin with membrane-mimicking interfacial monolayers}

\author{
Mehmet Nail Nasir ${ }^{1 凶}$, Emmanuel Benichou², Jean Sébastien Guez ${ }^{3}$, Philippe Jacques ${ }^{3}$, Pierre-François \\ Brevet $^{2}$, Françoise Besson ${ }^{1}$ \\ Institut de Chimie et Biochimie Moléculaires et Supramoléculaires, UMR CNRS 5246, Université Lyon \\ 1, Villeurbanne, France. \\ ${ }^{2}$ Laboratoire de Spectrométrie Ionique et Moléculaire, UMR CNRS 5579, Université Lyon 1, \\ Villeurbanne, France. \\ ${ }^{3}$ Laboratoire de Procédés Biologiques, Génie Enzymatique et Microbien (ProBioGEM, UPRES EA 1026), \\ Polytech'Lille, IUT A, Université Lille Nord de France, USTL, Villeneuve d'Ascq Cedex, France.
}

Email: nasir@univ-lyon1.fi 


\begin{abstract}
Mycosubtilin is a strong antimicrobial agent belonging to the iturinic lipopeptide family which contains a single tyrosine residue. Its cell target has been shown to be the cytoplasmic membrane. This tyrosine residue has been previously shown to be essential for the biological activity of mycosubtilin. Since we have previously demonstrated that tyrosine, an aromatic amino acid, can be used as an endogenous probe for the frequency doubling process, the presence of a tyrosine residue in mycosubtilin allowed us to investigate the interactions of mycosubtilin with biomimetic lipid monolayers at the air-water interface by Second Harmonic Generation (SHG). Mycosubtilin was added underneath dipalmitoylphosphatidylcholine (DPPC) or cholesterol monolayers at the air-water interface and significant increases in the surface pressure were observed in both cases. This observation demonstrates that mycosubtilin interacts with these biomimetic membranes. A light polarization resolved analysis of the SHG signals recovered for these two systems was then performed and confirmed that those interactions between the tyrosine residue in mycosubtilin and the membranes could be monitored by SHG. Furthermore, the differences exhibited by the nonlinear optical measurements for different membranes showed that these interactions depend on the nature of the biomimetic membrane present at the air-water interface.
\end{abstract}

Key words. mycosubtilin, biomimetic membranes, second harmonic generation, iturin, antimicrobial peptide 


\section{Introduction}

Second Harmonic Generation (SHG) is the nonlinear optical process whereby two photons at a fundamental frequency are converted into one photon at the harmonic frequency. SHG has been shown to be an ideal tool to investigate molecular organization at the nanometer scale owing to its cancellation in centrosymmetric media like liquids within the electric dipole approximation. Hence, SHG has been applied in the past for the characterization of many different systems at liquid interfaces $[1,2]$. Besides, recent studies aiming at extending its use to proteins and peptides in the absence of exogenous molecular probes have been recently performed. In particular, they demonstrated that aromatic amino acids like tryptophan (Trp) and tyrosine (Tyr) can be used as endogenous probes of proteins and peptides. The first hyperpolarizability of Trp and Tyr, namely their cross-section for the SHG process, and that of a short tripeptide containing two lysines and one Trp, were indeed determined by Hyper Rayleigh Scattering (HRS), an incoherent SHG process, in the bulk of an aqueous phase [3]. These measurements therefore showed that it is possible to devise experiments targeting Trp and Tyr residues in short synthetic peptides using SHG at the air-water interface $[4,5]$. These results confirmed the previous works reported for Trp derivative at interfaces and extended them to the case of Tyr [6, 7]. As a result, through a detailed analysis of the nonlinear optical response, in particular when selecting the polarization state of the incoming and outgoing fields, a molecular picture of the interface can be retrieved, a powerful tool to investigate the interaction of Trp- and Tyr-rich peptides with phospholipids [2, 8].

In the present work, we perform SHG experiments probing at the air-water interface the presence of a biologically-active peptide containing a single Tyr residue. Indeed, mycosubtilin is constituted by the heptapeptide L-Asn $\rightarrow$ D-Tyr $\rightarrow$ D-Asn $\rightarrow$ L-Gln $\rightarrow$ L-Pro $\rightarrow$ D-Ser $\rightarrow$ L-Asn associated with a $\beta$-amino fatty acid residue [9]. Mycosubtilin is a strong antimicrobial cyclolipopeptide produced by Bacillus subtilis strains and involved in the biological control of plant diseases [10-11]. Its cell target has been shown to be the cytoplasmic membrane [12]. To model the interaction between the lipopeptide and the biological membranes, its interfacial properties and its adsorption to various lipid monolayers at the air-water interface have been extensively studied and it has been shown that cholesterol is a preferential partner of mycosubtilin when it interacts with biomimetic membranes [13-15]. We therefore investigated the 
adsorption of mycosubtilin to interfacial monolayers constituted by DPPC or cholesterol by simultaneously measuring the SHG signal and the surface pressure by standard tensiometry.

\section{Materials and methods}

\subsection{Chemicals.}

Cholesterol (Chol) and 1,2-dipalmitoyl-sn-glycero-3-phosphocholine (DPPC), purchased from Sigma Chemical (St. Louis, MO), were dissolved in hexane-ethanol $(9 / 1, \mathrm{v} / \mathrm{v})$. Mycosubtilin, prepared as described previously [16], was dissolved in dimethylsulfoxide.

\subsection{Film formation and surface pressure measurements.}

Adsorption experiments were performed at $18^{\circ} \mathrm{C}$ using a small Teflon dish ( $4 \mathrm{~cm}$ diameter, $12 \mathrm{~mL}$ volume) and a pure water subphase (resistivity of $18.2 \mathrm{M} \Omega \cdot \mathrm{cm}$ ). This low temperature was required for the optical experiments to prevent evaporation during the course of the measurements. The lipid monolayers were prepared by deposition of the DPPC or Chol solutions at the air-water interface, thereby setting an initial surface pressure Пi of $10 \mathrm{mN} / \mathrm{m}$. After stabilization of the lipid monolayer, mycosubtilin was injected into the subphase at a final concentration of $1.1 \mu \mathrm{M}$. To avoid fluctuations in the SHG optical measurements, the subphase was stirred only during the first minutes after the lipopeptide injection.

\subsection{Second Harmonic Generation measurements}

The SHG setup was based on a femtosecond Ti-sapphire oscillator laser source providing pulses with duration of about $70 \mathrm{fs}$ at a repetition rate of $80 \mathrm{MHz}$ (Spectra-Physics) [17, 18]. After passing through a low-pass filter to remove any unwanted harmonic light generated prior to the interface, the fundamental beam set to a wavelength of $800 \mathrm{~nm}$ and an averaged power of about $1 \mathrm{~W}$ was focused by a lens with a 10 $\mathrm{cm}$ focal length onto the air-water interface. The incidence angle was set at a value of $70^{\circ}$ corresponding to an optimum incidence angle for the SHG intensity in reflection. The SH light was collected by a lens with a $10 \mathrm{~cm}$ focal length and separated from its fundamental counterpart by a high-pass filter. The SH light was detected with a water-cooled back-illuminated CCD camera (Andor) placed after a spectrometer (JobinYvon). The fundamental input beam was linearly polarized and the input polarization angle $\gamma$ was selected 
with a rotating half-wave plate. The angle $\gamma=0$ corresponds to a p-polarized fundamental beam and $\gamma=\pi / 2$ to an s-polarized fundamental beam. An analyzer, placed in front of the spectrometer, was used to separate the S- and P-polarized SH intensities. In all the kinetic experiments, we used a p-in and P-out polarization configuration.

\section{Results and discussion}

\subsection{Interaction of mycosubtilin with the DPPC monolayer}

The interactions between mycosubtilin and the phospholipids were analyzed using DPPC as a model phospholipid. An initial surface pressure $\Pi i$ of $10 \mathrm{mN} / \mathrm{m}$ was chosen for the DPPC monolayer since it has been previously shown that mycosubtilin induces a significant increase in the surface pressure $\Delta \Pi$ for this initial pressure Пi [14]. Before the mycosubtilin injection, the SHG signal of the DPPC monolayer at 10 $\mathrm{mN} / \mathrm{m}$ was about one and a half times higher than that obtained for the neat air-water interface. After the mycosubtilin injection underneath the DPPC monolayer, the adsorption kinetics of the lipopeptide to the phospholipid monolayer was followed through the simultaneous measurements of the SHG signal and the surface pressure. The kinetics of the mycosubtilin-induced changes in the SHG signal and the surface pressure are shown in Fig. 1. The surface pressure increases gradually and then reaches a plateau corresponding to a surface pressure change $\Delta \Pi$ of about $15 \mathrm{mN} / \mathrm{m}$. This indicates that mycosubtilin adsorbs to the interface. The SHG signal increased faster than the surface pressure until a plateau was also reached. To get further information on the molecular organization and/or orientation of the species present at the airwater interface, SHG light polarization curves were recorded for the DPPC monolayer in the absence and in the presence of mycosubtilin, see Fig. 2. The graph obtained for the pure DPPC monolayer at $10 \mathrm{mN} / \mathrm{m}$, see Fig. 2A, is very similar to that obtained for the neat air-water interface (data not shown). The P-polarized curve has two maxima while the S-polarized one has four maxima. The maximum intensity of the Ppolarized curve is about three times higher than that measured for the S-polarized one. The $45^{\circ}$-polarized curve has two maxima with intermediate intensity. The latter curve is required besides the P- and Spolarized ones to lift a phase ambiguity on the susceptibility tensor elements, see below. Light polarization curves of the DPPC monolayer were also recorded after complete adsorption of the lipopeptide (i.e. when the surface pressure reaches the plateau). The P-polarized curve still exhibits two maxima, as it was 
observed with the neat air-water interface and the pure DPPC monolayer. However, the presence of mycosubtilin in the DPPC monolayer decreases significantly the intensity of the maxima of the S-polarized curve, see Fig. 2B. Thus, the ratio of the intensity maxima of the P-and S- polarized curves reaches about 10 while it was only about 3.5 in the case of pure DPPC monolayer. As a consequence, a smaller shift of the $45^{\circ}$-polarized curve is observed as compared to the shift in the absence of mycosubtilin. These data therefore confirm that the interaction of mycosubtilin with the phospholipid membrane is indeed monitored. In order to quantify this interaction, the P-polarized and S-polarized plots can be analyzed using the standard form of the SH intensity in the electric dipole approximation as a function of the input polarization angle $\gamma[17]$ :

$$
\begin{aligned}
& I_{s}(\gamma) \propto\left|a_{1} \chi_{x x z} \sin (2 \gamma)\right|^{2} \\
& I_{p}(\gamma) \propto\left|\left(a_{2} \chi_{x x z}+a_{3} \chi_{z x x}+a_{4} \chi_{z z z}\right) \cos ^{2} \gamma+a_{5} \chi_{z x x} \sin ^{2} \gamma\right|^{2}
\end{aligned}
$$

where the $a_{i}(i=1-5)$ are five constants depending on the geometrical configuration and the optical indices of water and air at $800 \mathrm{~nm}$ and $400 \mathrm{~nm}$. The quantities $\chi_{z z z}, \chi_{z x x}$ and $\chi_{x x z}$ are the three non vanishing and independent quadratic susceptibility tensor components associated with an isotropic and achiral interface in the electric dipole approximation. Using Eqs.(1) to fit the experimental data, we obtain the values for the ratios $\chi_{z x x} / \chi_{z z z}$ and $\chi_{x x z} / \chi_{z z z}$. These values are given in Table 1 . For the pure DPPC monolayer, the results are similar to those obtained in the case of a neat air/water interface, as reported previously [19]. These ratios however dramatically drop in the presence of mycosubtilin demonstrating the occurrence of interactions between the peptide and the lipid membrane. The signature of this interaction is a reinforcement of the out-of-plane contribution, namely the $\chi_{z z z}$ element, of the non linear optical response.

\subsection{Interaction of mycosubtilin with cholesterol monolayer}

The interactions between mycosubtilin and sterols were analyzed using cholesterol as a model sterol. In a first step, we measured the SHG signal of a pure Chol monolayer at a surface pressure of $10 \mathrm{mN} / \mathrm{m}$. The SHG intensity was about three times higher than the SHG signal of the neat air-water interface and about 
two times higher than that of a pure DPPC monolayer at a surface pressure of $10 \mathrm{mN} / \mathrm{m}$. This can be related to the high packing of the Chol monolayer. After mycosubtilin injection, we simultaneously measured the SHG signal and the surface pressure, see Fig. 3. A weak increase of the SHG intensity was observed immediately after the lipopeptide injection, while the surface pressure increase occurred later. This latter increase was also more significant, with a value $\Delta \Pi$ of about $35 \mathrm{mN} / \mathrm{m}$. Such a time difference between the SHG increase and the surface pressure was also observed above with the DPPC monolayer. Furthermore, while the mycosubtilin-induced increase of the Chol monolayer SHG signal was weak, $\triangle \mathrm{SHG} \sim 0.7$ a.u., as compared to its initial value of $\sim 3$ a.u., this increase is similar to that observed with the DPPC monolayer, $\Delta \mathrm{SHG} \sim 0.6$ a.u.

The light polarization curves of the Chol monolayer in the absence and in the presence of mycosubtilin were then recorded, see Fig. 4. Without taking into account the different intensities, the light polarization curves of the pure Chol monolayer, see Fig. 4A, were very similar to those of the neat air-water interface (data not shown). The light polarization curves of the mixed Chol-mycosubtilin monolayer were then recorded after complete adsorption of the lipopeptide, see Fig. 4B. These curves are radically different from those of the pure Chol monolayer. This noteworthy difference between the light polarization curves of the Chol-mycosubtilin and pure Chol monolayers is observed in the practically complete disappearance of the S-polarized curve and the appearance of two weak peaks in the P-polarized curve at the fundamental polarization angles $90^{\circ}$ and $270^{\circ}$.

To test whether these changes are the signature of the interaction of the lipopeptide with cholesterol, mycosubtilin was injected under the Chol monolayer at surface pressures of $3 \mathrm{mN} / \mathrm{m}$ and $30 \mathrm{mN} / \mathrm{m}$. Light polarization curves of the Chol-mycosubtilin monolayers were then recorded after complete adsorption of the lipopeptide, i.e. when the surface pressure change $\Delta \Pi$ was stabilized at $\sim 25 \mathrm{mN} / \mathrm{m}$ for the surface pressure $\Pi$ i of $3 \mathrm{mN} / \mathrm{m}$ or stabilized at $\sim 10 \mathrm{mN} / \mathrm{m}$ for the surface pressure $\Pi$ i of $30 \mathrm{mN} / \mathrm{m}$. In both cases, the P-polarized curves exhibited the two weak peaks at the fundamental polarization angles $90^{\circ}$ and $270^{\circ}$ besides the two maxima obtained at 0 and $180^{\circ}$ (data not shown). In both cases, the complete disappearance of the S-polarized curve was observed. Using Eqs. (1), the elements of the susceptibility tensor were 
determined in the case of a pure cholesterol monolayer and a mixed mycosubtilin-cholesterol monolayer, see Table 1. For the pure cholesterol monolayer, the extracted value for the $\chi_{z x x} / \chi_{z z z}$ ratio is similar to that obtained in the case of a DPPC monolayer whereas a greater value is obtained for the $\chi_{x x z} / \chi_{z z z}$ ratio. As for the DPPC monolayer, the presence of mycosubtilin induced a dramatic drop of the two elements $\chi_{z x x}$ and $\chi_{x x z}$. The $\chi_{x x z}$ element vanishes as expected for a vanishing S-polarized intensity curve. Similarly, the absolute value of the ratio $\chi_{z x x} / \chi_{z z z}$ also decreased as compared to the value obtained in the case of the pure cholesterol monolayer. However, this time the decrease is accompanied by a relative phase change. The latter change is associated with the response of the Tyr residue.

In conclusion, we have demonstrated that, while the SHG signals from the DPPC and Chol monolayers have not the same intensity, both give similar SHG light polarization curves. This similarity was not preserved when mycosubtilin interacted with the DPPC and the cholesterol monolayers. The mycosubtilininduced changes in the SHG light polarization curves are however more pronounced in the case of a Chol monolayer as compared to the case of the DPPG monolayer. Hence, SHG is demonstrated to be a powerful tool to investigate Tyr containing peptides, similarly to Trp containing peptides. It is also sensitive enough to monitor peptides containing a single Tyr residue. Further work will be carried on to extract the molecular parameters from these results and conclude further on these interactions. 


\section{References}

[1]. Shen, Y. R. (1986) Surface Second Harmonic Generation: A New Technique for Surface Studies. Ann. Rev. Mater. Sci. 16, 69-86.

[2]. Eisenthal, K. B. (1996) Liquid interfaces probed by second-harmonic and sum-frequency spectroscopy. Chem. Rev. 96 (4) 1343-1360

[3]. Duboisset, J., Matar, G., Russier-Antoine, I., Benichou, E., Bachelier, G., Jonin Ch., Ficheux, D., Besson, F., Brevet, P.F. (2010) First Hyperpolarizability of the Natural Aromatic Amino Acids Tryptophan, Tyrosine and Phenylalanine and the tripeptide Lysine - Tryptophan - Lysine Determined by Hyper Rayleigh Scattering, J. Phys. Chem. B., 114 (43) 13861-13865.

[4]. Corn, R. M., Higgins, D. A. (1994) Optical Second Harmonic generation as a probe of surfacechemistry. Chem. Rev. 94 (5) 107-125.

[5]. Shen, Y. R. (1984) The Principles of Nonlinear Optics, Wiley, New York.

[6]. Salafsky, J. S., Cohen, B. (2008) A Second-Harmonic-Active Unnatural Amino Acid as a Structural Probe of Biomolecules on Surfaces. J. Phys. Chem. B 112 (47) 15103-15107.

[7]. Mitchell, S. A. (2006) Origin of second harmonic generation optical activity of a tryptophan derivative at the air/water interface. J. Chem. Phys. 125 (4) 44716.

[8]. Matar, G., Duboisset, J., Benichou, E., Bachelier, G., Russier-Antoine, I., Jonin, C., Ficheux, D., Brevet, P. F., Besson, F. (2010) Second Harmonic Generation, a New Approach for Analyzing the Interfacial Properties of a Tryptophan-Rich Peptide. Chem. Phys. Lett. 500 (1-3) 161-166.

[9]. Peypoux, F., Pommier, M. T., Marion, D., Ptak, M. Das, B. C., Michel G. (1986) Revised structure of mycosubtilin, a peptidolipid antibiotic from Bacillus subtilis. J. Antibiot. 39 (5) 636-641.

[10] Leclere, V., Bechet, M., Adam, A., Guez, J. S., Wathelet, M., Ongena, M., Thonart, P., Gancel, F., Chollet-Imbert, M., Jacques, P. (2005) Mycosubtilin overproduction by Bacillus subtilis BBG100 enhances the organism's antagonistic and biocontrol activities. Appl. Environ. Microbiol. 71 (8) 4577-84.

[11] Ongena, M., Jacques, P. (2008) Bacillus lipopeptides : versatile weapons for plant disease biocontrol. Trends Microbiol. 16 (3) 115-125.

[12]. Besson, F., Michel G. (1989) Action of mycosubtilin, an antifungal antibiotic of Bacillus subtilis, on the cell membrane of Saccharomyces cerevisiae. Microbios 59 (239) 113-121. 
[13]. Maget-Dana, R., Ptak, M. (1990) Iturin lipopeptides: interactions of mycosubtilin with lipids in planar membranes and mixed monolayers. Biochim. Biophys. Acta 1023 (1) 34-40.

[14]. Nasir, M. N., Besson, F. (2011) Specific interactions of mycosubtilin with cholesterol-containing artificial membranes. Langmuir 27 (17) 10785-10792.

[15]. Nasir, M. N., Thawani, A., Kouzayha, A., Besson, F. (2010) Interactions of the natural antimicrobial mycosubtilin with phospholipid membrane models. Colloids Surf. B 78 (1) 17-23.

[16]. Besson, F., Peypoux, F., Michel, G., Delcambe, L. (1978) Identification of antibiotics of iturin group in various strains of Bacillus subtilis. J. Antibiot. 31 (4) 284-288.

[17]. Martin-Gassin, G., Benichou, E., Bachelier, G., Russier-Antoine, I., Jonin, Ch., Brevet, P. F. (2008) Compression induced chirality in dense molecular films at the air-water interface probed by second harmonic generation. J. Phys. Chem. C 112 (10) 12958-12965.

[18]. Rinuy, J., Brevet, P. F., Girault, H. H. (1999) Second harmonic generation of glucose oxidase at the air/water interface. Biophys. J. 77 (6) 3350-3355.

[19] Benichou, E., Martin-Gassin, G., Derouet, A., Russier-Antoine, I., Bachelier, G., Jonin, C., Lascoux, N., Liu, M., Brevet, P. F. (2011) Chirality in molecular films at the air-water interface. SPIE Proc. 7935, $79350 V-7935$. 


\section{Figure Captions}

Fig. 1 Combined tensiometry (dash line) and SHG (solid line) intensity time dependence during the mycosubtilin adsorption for a DPPC monolayer at the air-water interface. The SHG intensity is normalized to the neat air-water interface. Time zero corresponds to the lipopeptide injection under the DPPC monolayer at a surface pressure of $10 \mathrm{mN} / \mathrm{m}$. The final concentration of mycosubtilin in the pure water subphase was $1.1 \mu \mathrm{M}$

Fig. 2 SHG intensity as a function of the input polarization angle for a pure DPPC (a) and a mixed DPPCmycosubtilin (b) monolayer. The SHG intensity is normalized to the neat air-water interface. P-polarized SHG intensity corresponds to $(\bullet)$, S-polarized SHG intensity to $(\boldsymbol{\bullet})$ and $45^{\circ}$-polarized to ( $\left.\mathbf{(}\right)$

Fig. 3 Combined tensiometry (dash line) and SHG (solid line) intensity time dependence during the mycosubtilin adsorption for a Chol monolayer at the air-water interface. The SHG intensity is normalized to the neat air-water interface. Time zero corresponds to the lipopeptide injection under the Chol monolayer at a surface pressure $10 \mathrm{mN} / \mathrm{m}$. The final concentration of mycosubtilin in the pure water subphase was 1.1 $\mu \mathrm{M}$

Fig. 4 SHG signal intensity as a function of the input polarization angle for a pure Chol (a) and a mixed Chol-mycosubtilin (b) monolayers. The SHG intensity is normalized to the neat air-water interface. Ppolarized SHG intensity corresponds to $(\bullet)$, S-polarized SHG intensity to $(\bullet)$ and $45^{\circ}$-polarized to ( $\left.\square\right)$ 
table1

Click here to download table: Table 1.docx

Table 1 Values obtained for the $\chi_{x x z} / \chi_{z z z}$ and $\chi_{z x x} / \chi_{z z z}$ ratios from the adjustment to Eqs.(1).

\begin{tabular}{l|lllll}
\hline & $\begin{array}{l}\text { Neat } \\
\text { air/water } \\
\text { interface }\end{array}$ & $\begin{array}{l}\text { Pure DPPC } \\
\text { monolayer }\end{array}$ & $\begin{array}{l}\text { Mixed MS- } \\
\text { DPPC } \\
\text { Monolayer }\end{array}$ & $\begin{array}{l}\text { Pure } \\
\text { Cholesterol } \\
\text { monolayer }\end{array}$ & $\begin{array}{l}\text { Mixed MS- } \\
\text { cholesterol } \\
\text { monolayer }\end{array}$ \\
\hline$\chi_{x x z} / \chi_{z z z}$ & 0.38 & 0.32 & 0.19 & 0.32 & 0.00 \\
$\chi_{z x x} / \chi_{z z z}$ & 0.08 & 0.10 & 0.02 & 0.21 & -0.11 \\
\hline
\end{tabular}

MS, mycosubtilin. ${ }^{a}$, taken from ref.[19] 
line figure 2

Click here to download line figure: Figure2.eps
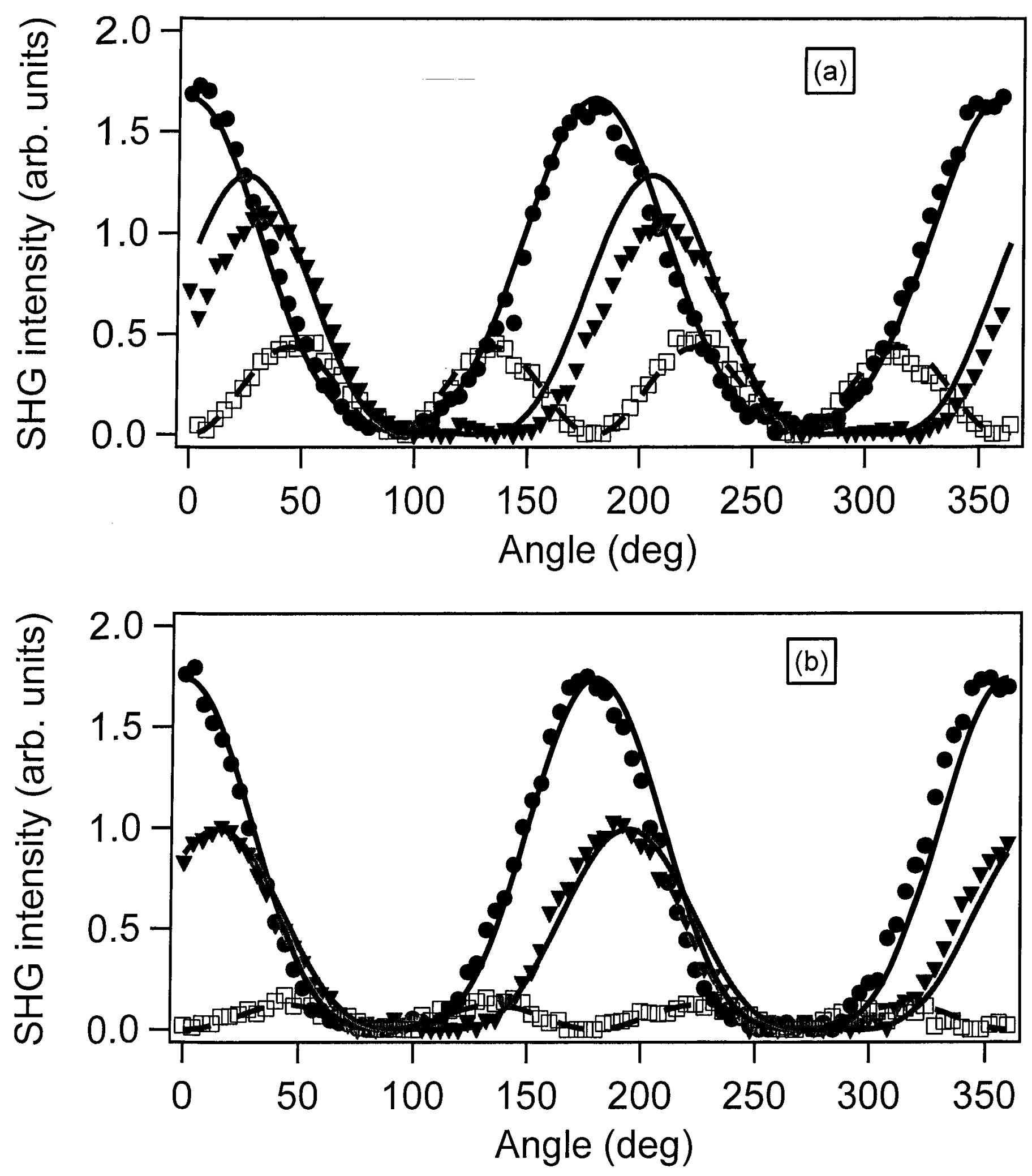
line figure 3

Click here to download line figure: Figure3.eps

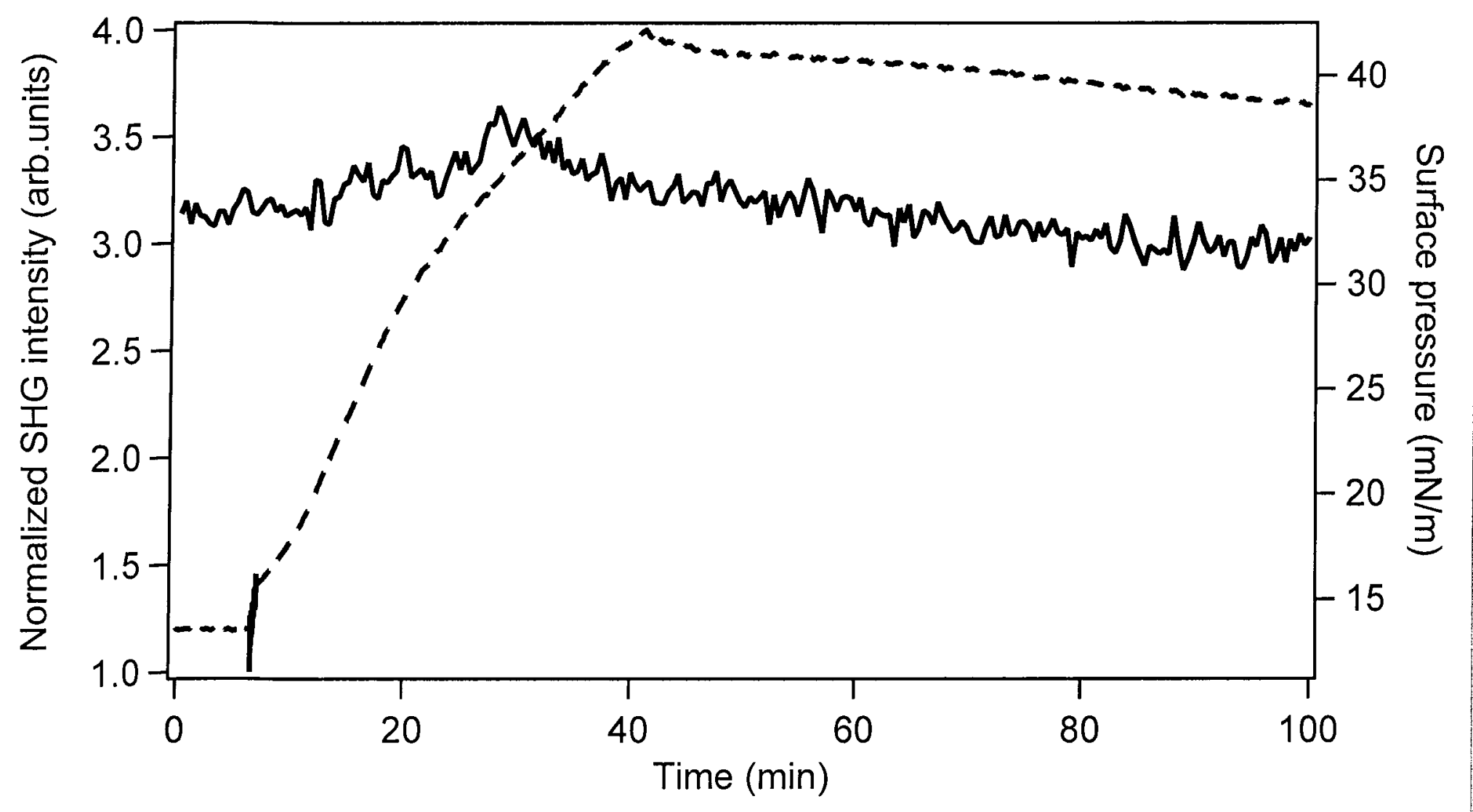


line figure 4

Click here to download line figure: Figure4.eps
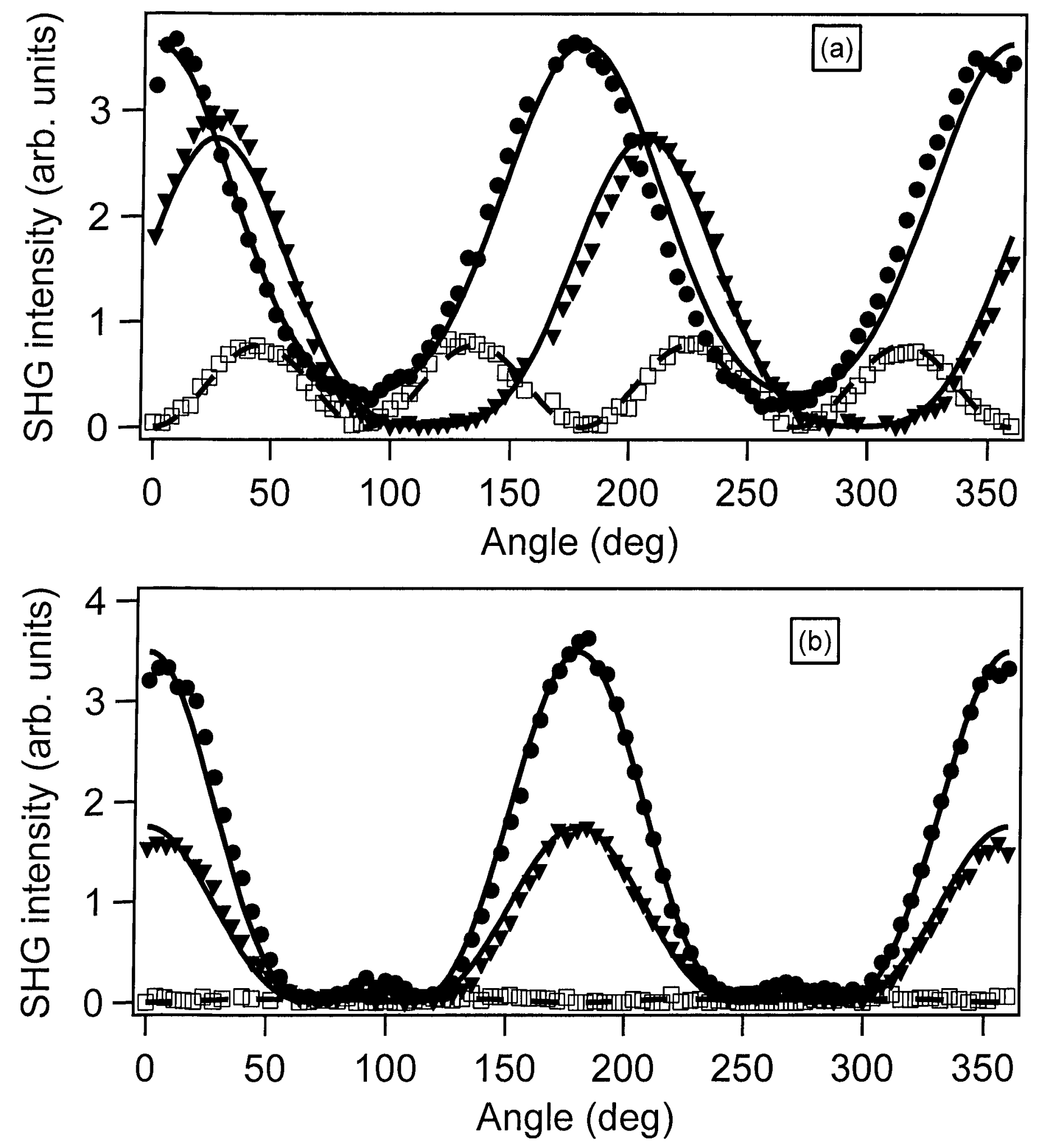
